\title{
Comparison of Round Window Anatomy between Pediatric and Adult Population: A Perspective from Cochlear Implantation Surgery
}

\author{
Rabindra B Pradhananga, Bigyan R Gyawali, Pabina Rayamajhi \\ Department of ENT, Head \& Neck Surgery, Maharajgunj Medical Campus, Ganesh Man Singh Memorial Academy \\ of ENT - Head \& Neck Studies, Tribhuvan University Teaching Hospital, Maharajgunj, Kathmandu, Nepal
}

\section{Corresponding author:}

\section{Bigyan R Gyawali, MS, MRCS}

Department of ENT, Head \& Neck Surgery, Maharajgunj Medical Campus, Ganesh Man Singh Memorial Academy of ENT - Head \& Neck Studies, Tribhuvan University Teaching Hospital, Maharajgunj, Kathmandu, Nepal Email: bigyan.gyawali@gmail.com

Submitted : July 7, 2020

Accepted : Nov 16, 2020

\begin{abstract}
\section{Introduction}

The round window is thought to be an ideal port for inserting electrodes during cochlear implantation. Considering its complex anatomy with an individual variation, this study aims to review the anatomy of round window based on the visibility of round window niche and round window membrane via posterior tympanotomy in pediatric and adult population who underwent cochlear implantation.

\section{Methods}

This was a retrospective observational study conducted at the Department of ENT-HNS, Institute of Medicine, Kathmandu, Nepal. Surgical notes of adult ( $>15$ years) and pediatric cases (<15years) who underwent primary cochlear implantation from January 2015 to January 2018 were assessed for different grading of round window niche and round window membrane visibility via posterior tympanotomy. Cases with revision surgery and with incomplete documentation of intra-operative findings were excluded from the study. Statistical analysis was done using SPSS software version 25. We used Chi-square and Fisher's exact tests to analyze the statistical association.
\end{abstract}

\section{Results}

Type $B$ round window niche (partially visible) was the most common variant seen in the pediatric group while in adults, both Type B (partially visible) and Type C (fully visible) round window niche were common. Compared to the adults, the pediatric group had good visibility of RWM. However, there was no statistical association between these observations.

\section{Conclusion}

The round window has a wide range of anatomical variations with different levels of visibility of RWN and RWM in the different age groups. Although statistically insignificant, RWM visibility seemed to be better in pediatric cases compared to adults.

\section{Keywords}

Anatomical variation, cochlear implantation, round window 


\section{INTRODUCTION}

C ompared to the earlier years, indications today have broadened for cochlear implantation. Although a commonly practiced surgery in the present context, the complexity of the anatomy of the temporal bone often renders the technicality of the procedure difficult. A range of approaches to access inner ear are described such as facial recess approach, suprameatal, transcanal, pericanal and middle cranial fossa approach. ${ }^{1-3}$ Whichever may be the approach, round window (RW) remains the most favored port for entry to the inner ear, considering a less traumatic procedure with preservation of hair cells. ${ }^{4,5}$

The surgeon should be very well oriented with the anatomy of RW which usually differs in different age groups. The rationale of our study was thus to evaluate the anatomical variations of RW in terms of visibility of round window niche (RWN) and round window membrane (RWM) in both adult and pediatric populations.

\section{METHODS}

This retrospective observational study was conducted at the Department of ENT-HNS, Tribhuvan University Teaching Hospital, Institute of Medicine, Kathmandu, Nepal. Approval from the Institutional Review Committee was taken. Surgical notes of all the adult cases ( $>15$ years) who underwent primary Cl from January 2015 to January 2018 were included in the study. The similar number of pediatric cases ( $<15$ years) who underwent $\mathrm{Cl}$ within the same frame of time were also selected randomly by lottery method to have an equal number of the comparison group. Cases with revision surgery and with an incomplete documentation of intra-operative findings were excluded from the study. We looked for different grading of RWN and RWM visibility via the posterior tympanotomy and to compare the RW variations between the two groups.

Round window membrane (RWM) visibility was classified as follows: 6

- Grade I : $>50 \%$ of RWM is visible

- Grade II : 25\%-50\% of RWM is visible

- Grade III : $<25 \%$ of RWM is visible

- Grade IV : RWM is not visible

Round window niche (RWN) visibility was classified as follows: ${ }^{7}$

- Type A : Difficult to visualize

- Type B : Partially visible

- Type C : Fully visible

Statistical analysis was done using SPSS software version 25. Chi-square and Fisher's exact tests were used to analyze the statistical association.

\section{RESULTS}

A total of 20 cases were included in this study with ten in the adult group and ten in the pediatric group. Male to female ratio was 2:1. The majority of pediatric cases were between three to six years of age. Similarly, in the adult group, the majority of cases were in their third to six decades (Table 1).

Table 1. Age distribution $(n=20)$

\begin{tabular}{lc}
\hline \multicolumn{1}{c}{ Age group } & Frequency \\
\hline Pediatric & \\
$<3$ years & 3 \\
3-6 years & 5 \\
$>6$ years & 2 \\
Adult & \\
$<30$ years & 4 \\
$30-60$ years & 5 \\
$>60$ years & 1 \\
\hline
\end{tabular}

In the pediatric group, Type B was the most common RWN. No case with Type A RWN was seen. On the contrary, in the adult group, two cases had Type A RWN. Type B and Type C RWN were seen in four cases each. There was no statistical significance of this distribution of RWN amongst the two groups (Table 2).

Table 2. Round window niche visibility among different age groups $(n=20)$

\begin{tabular}{|c|c|c|c|c|}
\hline \multirow{2}{*}{$\begin{array}{l}\text { Age } \\
\text { group }\end{array}$} & \multicolumn{3}{|c|}{$\begin{array}{c}\text { Round window } \\
\text { niche visibility type }\end{array}$} & \multirow[t]{2}{*}{$p$-value } \\
\hline & A & B & $C$ & \\
\hline Pediatric & 0 & 8 & 2 & 0.13 \\
\hline Adult & 2 & 4 & 4 & \\
\hline
\end{tabular}

Similarly, RWM visibility had a variable distribution among the pediatric group with five having Grade II, three having Grade IV and two having grade I RWM visibility. The majority of the adult cases had Type IV RWM. This pattern of distribution of RWM visibility also didn't yield any statistical significance (Table 3).

Table 3. Round window membrane visibility among different age groups $(n=20)$

\begin{tabular}{llllll}
\hline \multirow{2}{*}{$\begin{array}{c}\text { Age } \\
\text { group }\end{array}$} & \multicolumn{4}{c}{ Round window } & \\
\cline { 2 - 5 } & membrane visibility grade & p-value \\
\cline { 2 - 5 } & I & II & III & IV & \\
\hline Pediatric & 2 & 5 & 0 & 3 & 0.31 \\
Adult & 1 & 2 & 1 & 6 & \\
\hline
\end{tabular}




\section{DISCUSSION}

With the development of new atraumatic electrode arrays and the concept of residual hearing preservation, the RW approach has gained huge popularity at current period of time. For cochlear implantation, every surgeon must be well oriented to the anatomy of RW as the RW is considered the key port of entry to the inner ear during implantation of the electrodes. Considering a wide range of anatomic variations of the RW, this study aimed to review and compare its anatomy based on RWN and RWM visibility via posterior tympanotomy in pediatric and adult population.

The majority of the pediatric cases in our study were above 3 years of age. Although implantation is recommended before 3 years of age, lack of awareness, level of education, economic and geographical constraints are the factors in our part of the world, that influence this delayed presentation and thus, the delayed intervention. The children even implanted after 3 years of age may still benefit from $\mathrm{Cl}$, as shown by the study of Gaurav et al. ${ }^{8}$ In adults, the majority were in their $3^{\text {rd }}$ to $6^{\text {th }}$ decades of life.

The round window has a complex anatomy. Its ossification is found to begin in the $16^{\text {th }}$ week of life and gets completed by birth. The anterosuperior and posteroinferior niche walls develop from intramembranous and enchondral ossification respectively. The differential growth of these walls tend to determine the shape of RWN. ${ }^{9}$ Different shapes of the RW have been described in the literature such as saddle, oval, round, triangular, comma, quadrangular, pear shaped, etc. ${ }^{10}$ The RW opening is usually directed posteriorly, inferiorly or posteroinferiorly. The more posterior and inferior the location of RW, the more is the difficulty in visualizing the RW via the facial recess. Several studies have been done to classify the RWN and RWM visibility via the facial recess approach. ${ }^{6,7}$ In our study, RWN with different levels of visibility had a varied distribution amongst both groups, however, Type A RWN (not visible) was only encountered in the adult population. RWM visibility, on the contrary, had a different picture. While the majority of the cases in the adult population had poor RWM visibility, RWM in the pediatric cases, on the other hand, seemed to have good visibility compared to the adults. This observation of ours couldn't yield any statistical significance. In all cases with poor visibility of RWN, RWM visibility also decreases. However, in cases with a fully visible RWN, RWM visibility will however, depend on the size of anterior and posterior bony overhangs of RW. Results from the study by Kashio et al. were contradictory to ours. ${ }^{7}$ The majority of the adults had fully visible RWN (44.3\%) followed by partially visible and invisible RWN. On the other hand, the majority of the children had (35.6\%) had partially visible RWN followed by invisible and then fully visible RWN. The observed difference also yielded statistical significance. Another comparative study by Panda et al. between the population of southern and northern India undergoing $\mathrm{Cl}$ showed Grade III RWM was the most common type of RWM in both populations. There was a significant difference in the distribution of other grades of RWM visibility i.e., grade I, II, and IV between two groups. Their study, however, didn't specify the age group. ${ }^{6}$ Dalmia et al. in their study, used a different classification for grading RWM visibility in the pediatric population. In contrast to our study, majority of the children $(60.53 \%)$ in their study had Type IV RWM visibility (25\%-49\%). Only in 5\% of the cases RWM was fully visible. ${ }^{11}$

Very low sample size was a major drawback of our study. Although grading of visibility of RWN and RWM was entirely subjective, involvement of a single operating surgeon minimized the observation bias. A study with a larger sample size should be considered further to yield more specific results.

\section{CONCLUSION}

The round window has a wide range of anatomical variations with different levels of visibility of RWN and RWM in the different age groups. Although statistically insignificant, RWM visibility seemed to be better in pediatric cases compared to adults.

\section{CONFLICT OF INTEREST}

None declared.

\section{REFERENCES}

1. House WF. Cochlear implants. Ann Otol Rhinol Laryngol. 1976;85 suppl 27(3Pt2):1-93.

2. El-Anwar MW, ElAassar AS, Foad YA. Non-mastoidectomy Cochlear Implant Approaches: A Literature Review. Int Arch Otorhinolaryngol. 2016;20(2):180184.

3. Mankekar G, Arriaga MA, Viator D, Volk JM. Cochlear implantation via middle fossa approach - a case report. Cochlear Implants Int. 2019;20(4):222-227.

4. Havenith S, Lammers MJ, Tange RA, et al. Hearing preservation surgery: cochleostomy or round window approach? A systematic review. Otol Neurotol 2013;34(4):667-674.

5. Khater A, El-Anwar MW. Methods of Hearing Preservation during Cochlearlmplantation. Int Arch Otorhinolaryngol. 2017;21(3):297-301.

6. Panda N, Kameswaran M, Patro SK, Saran S, Nayak G. Evaluation of Round Window Accessibility for Electrode Insertion: Validation Study from two Centers. J Otolaryngol-ENT Res. 2017;8(5).

7. Kashio A, Sakamoto T, Karino S, et al. Predicting round window niche visibility via the facial recess using high-resolution computed tomography. Otol Neurotol 2015;36(1):e18-23

8. Gaurav V, Sharma S, Singh S. Effects of Age at Cochlear Implantation on Auditory Outcomes in Cochlear Implant Recipient Children. Indian J Otolaryngol Head Neck Surg. 2020;72(1):79-85.

9. Tóth M, Alpár A, Patonay L, Oláh I. Development and surgical anatomy of the round window niche. Ann Anat.2006; 188:93-101.

10. Singla A, Sahni D, Gupta AK, Loukas M, Aggarwal A. Surgical anatomy of round window and its implications for cochlear implantation. Clin Anat. 2014; 27:331-6.

11. Dalmia D, Behera SK. Significance of round window niche drilling for cochlear implant surgery. Indian J Otol. 2017;23(3):141. 